\title{
Gap-Leaping Vortical Currents
}

\author{
N. RobB McDonald And E. R. JOHNSON \\ Department of Mathematics, University College London, London, United Kingdom
}

(Manuscript received 25 November 2008, in final form 3 April 2009)

\begin{abstract}
A one-parameter family of exact solutions describing the bifurcation of a steady two-dimensional current with uniform vorticity near a gap in a thin barrier is found. The unsteady evolution of source-driven flows toward these steady states is studied using a version of contour dynamics, appropriately modified to take into account the presence of a barrier with a single gap. It is shown that some of the steady solutions are realizable as large-time limits of the source-driven flows, although some are not owing to persistent eddy-shedding events in the vicinity of the gap. For the special case when there is zero net flux through the gap, numerical experiments show that the through-gap flux of vortical fluid increases with the width of the gap and that this flux approaches a steady limit with time.
\end{abstract}

\section{Introduction}

Circulation in the oceans is characterized by the presence of intense boundary currents. These vary from large-scale currents such as the Gulf Stream, deep western boundary currents, and the buoyancy-driven Leeuwin Current to smaller coastal flows driven by river outflow plumes under the influence of the Coriolis force. In many cases the boundaries along which these currents flow are not perfect barriers but instead are perforated by a series of gaps and straits. Many of the world's oceans and marginal seas are connected through such narrow passages. Examples include numerous island arcs in the ocean, including the Indonesian Archipelago connecting the Pacific and Indian Oceans and the Lesser Antilles, which forms a common boundary between the Caribbean and the tropical Atlantic. Additionally, the abyssal ocean can be thought of as a series of subbasins separated by steep midocean ridges, which are "leaky" in the sense that some interbasin flow is permitted through narrow fracture zones.

Gaps and straits between ocean basins play an important role in regulating interbasin volume transports and fluxes of quantities such as heat and salt. Oceanographers have long recognized the importance of gap

Corresponding author address: Robb McDonald, Department of Mathematics, University College London, Gower Street, London, WC1E 6BT, United Kingdom.

E-mail: robb@math.ucl.ac.uk zone regions and have conducted theoretical and observational studies of various dynamical processes associated with such regions. For example, Pedlosky (2001) has studied the behavior of incident Rossby waves on an idealized meridional barrier with gaps, demonstrating their transparency. A gap in the Lomonosov Ridge, connecting two main basins of the Arctic Ocean, controls deep water renewal in the region and has been the subject of a field study by Timmermans et al. (2005). Sheremet (2001), using numerical methods, has quantified the penetration of a viscous western boundary current through a gap in a meridional barrier and, subsequently, has performed related laboratory experiments (Sheremet and Kuehl 2007). Herbaut et al. (1998) showed that the bifurcation of the coastal current near the Strait of Sicily is consistent with linear Kelvin wave dynamics. Pratt and Spall (2003) have modeled linear barotropic wind-driven flow between basins separated by a "porous" ridge (i.e., one with many gaps) and obtained a differential equation whose solution determines the magnitude of the zonal flow through the ridge. Nof and Im (1985) constructed a model for the nonlinear flow of a buoyant current through a gap and applied it to the passage of the coastal current along the Alaskan coast through Unimak Pass.

Johnson and McDonald (2004a, 2005) have studied the motion of barotropic vortices in the presence of an infinite barrier perforated by either a single gap or two gaps. They obtained exact analytical expressions for the trajectories of point vortices and compared these to 


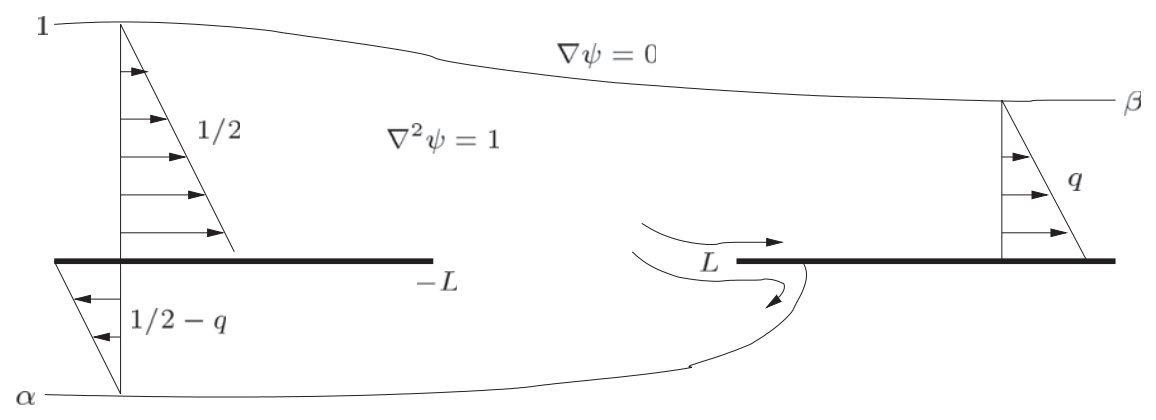

FIG. 1. Bifurcating vortical current near a gap. The arrows indicate the flow direction and their labels the nondimensional volume fluxes.

numerical computations of finite area patches of constant vorticity. In the case of point vortices, Crowdy and Marshall (2006) subsequently extended the results of Johnson and McDonald to barriers having an arbitrary number of gaps. Recently, Crowdy and Surana (2007) have detailed a method for implementing contour dynamics in domains with arbitrary connectivity, and this method could be used to study the motion of vortices near barriers with multiple gaps.

In this work, coastal currents are modeled as thin, two-dimensional, layers of inviscid fluid with anomalous (constant) vorticity surrounded by a larger ocean having zero vorticity. The anomalous vorticity of the current means that the layer of fluid will propagate parallel to the coastal barrier owing to the image effect (e.g., Stern and Pratt 1985; An and McDonald 2004, 2005). In this two-dimensional framework, the vorticity $\omega$ has the usual fluid dynamic definition $\omega=v_{x}-u_{y}$, where $(u, v)$ is the two-dimensional velocity field. This is equivalent to the potential vorticity for a homogeneous fluid of constant depth on the $f$ plane. While many coastal currents owe their origin to a balance between buoyancy and Coriolis forces, it is also the case that many do have anomalous vorticity and that this vorticity plays a role in their evolution (e.g., Kubokawa 1991). The assumption of constant vorticity means that the vorticity advection equation is satisfied trivially in the interior of the fluid, but the boundary separating regions of constant vorticity evolves in a complicated, nonlinear way and defines a difficult free boundary problem. It is, however, the assumption of constant vorticity that enables the use of powerful complex variable methods to find exact solutions for the structure of bifurcating currents near a gap (section 2) and allows the accurate computation of the evolution of such flows using a numerical (or semianalytical) method of contour dynamics (section 3). An important objective of the computations is to determine whether the exact steady solutions are realizable from an initial value problem in which a source of vortical fluid is initiated at $t=0$.

\section{Steady bifurcating vortical currents near a gap: Exact solutions}

\section{a. Problem formulation}

Consider a constant depth, homogeneous, and inviscid fluid. The two-dimensional velocity field $(u, v)$ can be derived from a streamfunction $\psi(x, y)$ via $u=-\psi_{x}$ and $v=\psi_{y}$, where $\nabla^{2} \psi=\omega$ is the vorticity of the fluid. A current, comprising fluid with constant vorticity $\omega_{0}$, propagates from $x=-\infty$ and approaches a gap centered on $x=0$ in an otherwise straight, infinitesimally thin, barrier. Outside the current the flow is irrotational; that is, $\nabla^{2} \psi=0$. Using the width of the current at $x=-\infty$ as the length scale and $\omega_{0}^{-1}$ as the time scale, the problem can be nondimensionalized, leading to the problem shown schematically in Fig. 1. Far from the gap the flow is parallel to the wall and therefore has velocity profile $u=1-y$. Thus, the incoming flux is $q=1 / 2$. It is assumed that the current bifurcates at the gap of nondimensional width $2 L$ such that its downstream thickness is $\beta$ with an associated flux $\beta^{2} / 2$, and the thickness of the current that passes through the gap approaches $|\alpha|$, where $\alpha<0$, with an associated flux $\alpha^{2} / 2$. Mass conservation gives

$$
\alpha^{2}+\beta^{2}=1
$$

Given $L$ and $\beta$, the aim is to find the shape of the boundary of the vortical current. Mathematically, this is a free boundary problem similar to those previously tackled by the authors (Johnson and McDonald 2006, 2007). Exact solutions can be found for the case when $u=v=0$ (or, equivalently, $\nabla \psi=0$ ) on the free boundary so that the fluid is everywhere stagnant outside the vortical current.

\section{b. Potential plane analysis}

To proceed, the problem shown in Fig. 1 is considered in the complex plane with the origin centered on the middle of the gap and the real axis aligned with the barrier. The velocity field can be written in complex form as 


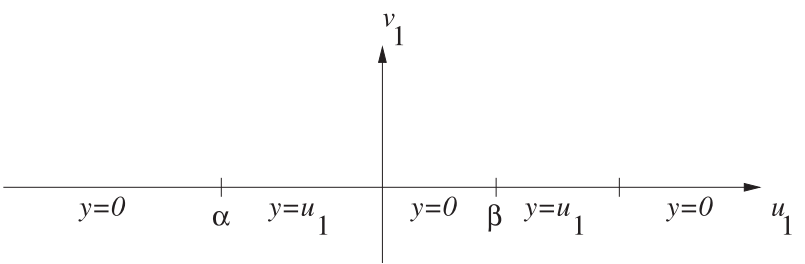

FIG. 2. The potential plane problem; $y$ satisfies Laplace's equation in the upper half of the $w$ plane.

$$
u-i v=-\frac{i}{2}[\bar{z}-S(z)]
$$

where $S(z)$ is an analytic function of $z$. The $-i \bar{z} / 2$ term on the rhs of (2) gives $v_{x}-u_{y}=1$ while the $S(z)$ term gives $v_{x}-u_{y}=0$ since it is analytic (and thus satisfies the Cauchy-Riemann equations). Hence, (2) implies that the velocity field has unit vorticity as required. Further, since $u=v=0$ on the free boundary, it follows that $S(z)=\bar{z}$ on the free boundary and is, therefore, the Schwarz function of the curve corresponding to the free boundary in the complex plane. Schwarz functions have been usefully employed in the study of other classes of flows with constant vorticity [e.g., finding families of vortex equilibria, Crowdy (1999)].

Define a new complex variable $w$ by

$$
w=u_{1}+i v_{1}=-\frac{i}{2}[z-S(z)]
$$

which, using (2), gives $u_{1}=y+u$ and $v_{1}=-v$. On the barriers and the free boundaries $v=0$; hence these are mapped to $v_{1}=0$ in the $w$ plane (i.e., the $u_{1}$ axis). Further, on the free boundaries $u_{1}=y$ since $u=0$. Hence $y=u_{1}$ on $v_{1}=0$ for $\alpha \leq u_{1} \leq 0$ and $\beta \leq u_{1} \leq 1$. For all other values on the $u_{1}$ axis the condition $y=0$ applies. The "potential" $w$ plane is shown in Fig. 2. Note that everywhere in the (physical) $z$ plane the flow is such that $v \leq 0$. Hence $v_{1} \geq 0$, and it follows that the flow is mapped to the upper half of the $w$ plane.

An analytic function $z=z(w)$ is sought in the $w$ plane such $\Im z=y$ has the behavior shown in Fig. 2 on the real $w$ axis. Elementary methods give

$$
z=C+\frac{w}{\pi} \log \left[\frac{w(w-1)}{(w-\beta)(w-\alpha)}\right],
$$

where $C$ is a real constant to be found. At $z=-L$ the velocity field must have inverse square root singularity that occurs at a $\pi$-radian corner (Howison and King 1989; Johnson and McDonald 2007); that is, $z \sim-L+w^{-2}$ as $w \rightarrow \infty$. Expanding (4) in the limit $w \rightarrow \infty$, using (1) and the condition at $z=-L$, gives

$$
C=-L+(1-\alpha-\beta) / \pi
$$

On the right-hand barrier $v=0$ there is a stagnation point where the vortical boundary detaches from the underside of the barrier as in Fig. 1. As $\Re z \rightarrow \infty$, the velocity becomes parallel to the barrier and, since it has unit vorticity, has the profile $u=\beta-y$. Therefore, in this limit, on the barrier $v=0$ and $u=\beta$. Restricting attention to the case in which $u$ is unidirectional on the right-hand barrier (i.e., $u \geq 0$ ), it follows that $u$ varies continuously in the interval $[0, \beta]$. The location of the tip of the right-hand barrier $z=L$ is then given implicitly as the smallest possible real value of $z$ that satisfies (4) for $0 \leq u<\beta$ (recall $w=u$ on the barrier); that is,

$$
L=C+0 \min _{0}\left\{\frac{u}{\pi} \log \left[\frac{u(u-1)}{(u-\beta)(u-\alpha)}\right]\right\} .
$$

Using (5) in (6) gives the gap width $2 L$ in terms of $\beta$ :

$$
\begin{aligned}
2 L= & \frac{1}{\pi}(1-\alpha-\beta) \\
& +0 \leq \min _{0}\left\{\frac{u}{\pi} \log \left[\frac{u(u-1)}{(u-\beta)(u-\alpha)}\right]\right\} .
\end{aligned}
$$

In summary, (1), (4), (5), and (7) define a one-parameter family of solutions for the free boundary shape. That is, choosing $0<\beta<1$, (1) gives $\alpha$, then (7) gives $L$ and (5) gives $C$, finally determining $z$ as a function of $u$ and $v$ through (4).

On the free boundary $w=y$ and (4) gives an explicit expression for the boundary of the current:

$$
x=-L+\frac{1}{\pi}(1-\alpha-\beta)+\frac{y}{\pi} \log \left[\frac{y(1-y)}{(y-\beta)(y-\alpha)}\right] .
$$

Streamlines of the flow field can be computed from (4) using the method described in Johnson and McDonald (2006). Figure 3 shows streamlines with evenly spaced values of the streamfunction for steady gap flows: $\beta=$ $0.80,0.50$, and 0.20 , corresponding to gap widths, determined from (6), $2 L=0.18,0.39$, and 0.55 , respectively. In steady flow of inviscid and incompressible fluid, the pressure $p$ can be calculated from the Bernoulli equation with $p / \rho+U^{2}$, where $\rho$ is the density and $U$ is the fluid speed, being constant along a streamline. The dashed line is the streamline that meets the tip of the barrier at $z=L$. The stagnation point on the right-hand barrier is shown by a cross. As expected, as the gap width decreases the across-gap flux increases. This is shown in Fig. 4 in which the across-gap current width $\beta$ is plotted against the gap width $2 L$. As $2 L \rightarrow 2 / \pi \approx 0.64$, the across-gap flux approaches zero and most of the current passes through the gap. This case is relevant to the flow of a coastal current about a sharp cape with the 

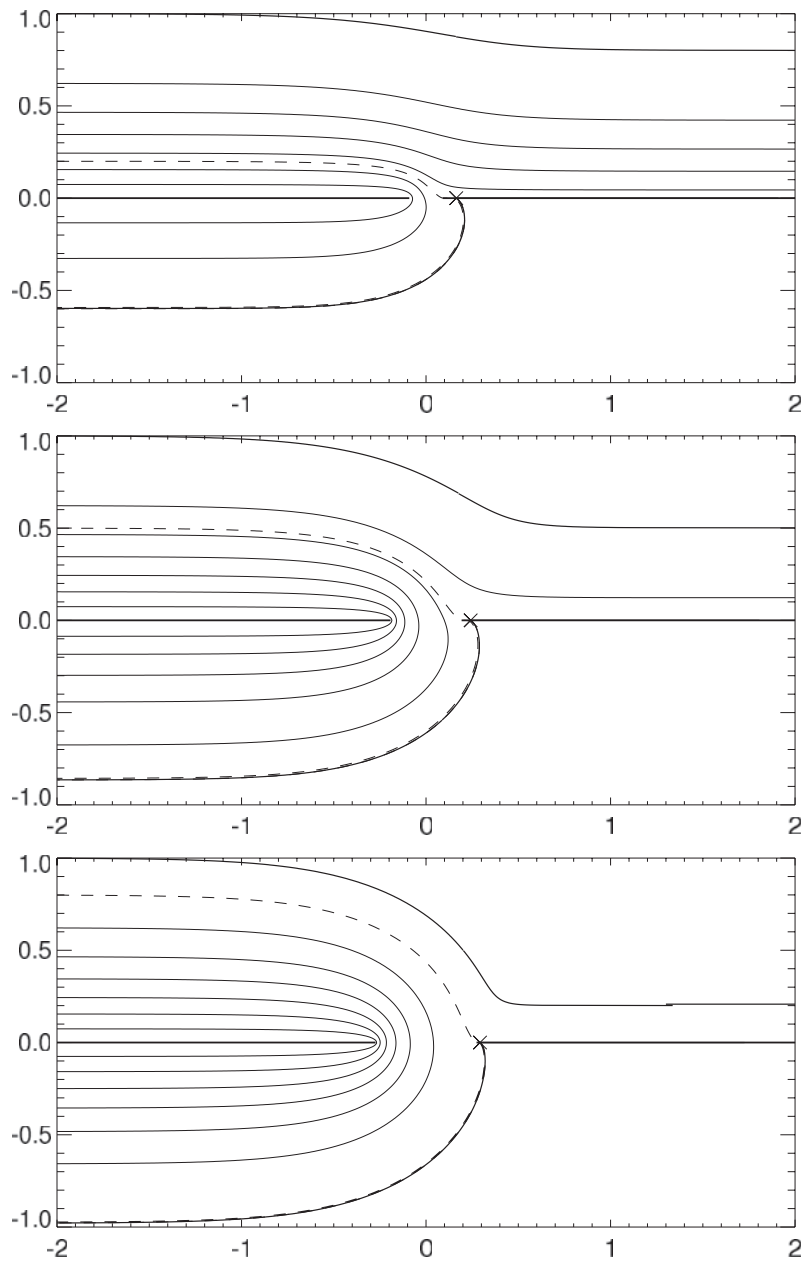

FIG. 3. Streamlines for steady gap flows: $\beta=0.8,0.4$, and 0.2 , corresponding to gap widths $2 L=0.18,0.39$, and 0.55 . The boundary of the current is shown as a thick solid line. Streamlines are at equally spaced values of the streamfunction. The dashed line is the streamline that meets the tip of the barrier at $z=L$. The stagnation point on the right-hand barrier is shown by a cross.

right-hand barrier playing no role in the steady dynamics since the flow is steady outside the vortical layer. It is noteworthy that this problem of finding a steady vortical current propagating around a semi-infinite barrier becomes mathematically equivalent to the finding the shape of a layer of viscous fluid coating a semi-infinite plate (a problem with industrial applications) for which the following exact solution exists (Howison and King 1989):

$$
x=\frac{2}{\pi}-\frac{y}{\pi} \log \left(\frac{1+y}{1-y}\right) .
$$

Note that (9) can be obtained from (8) (up to a translation in $x$ ) by putting $\beta=0$ and $\alpha=-1$. For gap widths

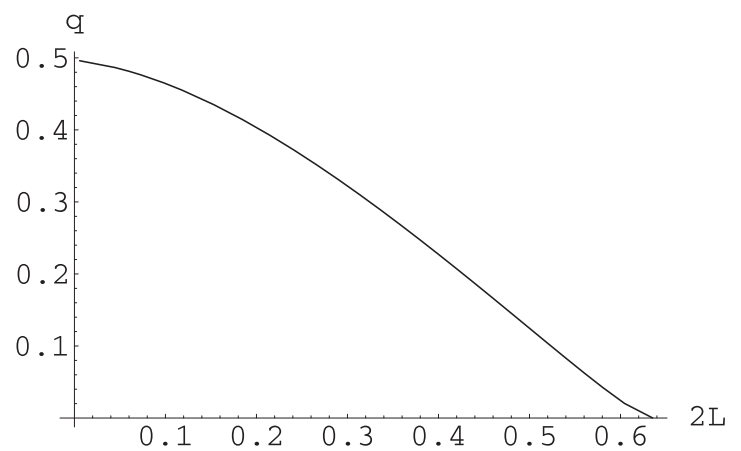

FIG. 4. Plot of across-gap flux $q$ as a function of gap width $2 L$.

greater than $2 / \pi$, all the current passes through the gap.

Since the length scale used to nondimensionalize the problem is the upstream current width $\left(2 q^{*} / \omega_{0}\right)^{1 / 2}$ where $q^{*}$ is the (dimensional) upstream flux and $\omega_{0}$ is the vorticity, the ratio of the gap width to upstream current width is $2 L /\left(2 q^{*} / \omega_{0}\right)^{1 / 2}$. In the limit $\omega_{0} \rightarrow 0$ the effective gap width vanishes and all of the current leaps across the gap.

\section{Computation of time-dependent flows}

It is of interest to determine if a time-dependent flow in which a coastal current encounters a gap evolves toward a member of the family of exact steady solutions derived above. The numerical method of contour dynamics provides an accurate method for studying the evolution of flows with piecewise-constant vorticity. It has been previously used to study the evolution and stability of coastal currents (e.g., Stern and Pratt 1985; Pratt and Stern 1986) and their interaction with topographic features such as shelves, canyons, and headlands (e.g., Cherubin et al. 1996; An and McDonald 2004, 2005). As in Johnson and McDonald (2006), the timedependent current is generated by a source in the wall, here located upstream of the gap at $z=-4$. Given that a steady-state current reaches its maximum width of unity exponentially quickly with distance downstream of the source (Johnson and McDonald 2006), locating the source at $z=-4$ is sufficiently far from the gap to be considered at "infinity." Equally, it is sufficiently close to the gap to enable the interaction of the current with the gap to be studied numerically in a reasonable time. As a check, numerical experiments for other choices of upstream source location were performed and little dependence on the source location was evident. The contour dynamics algorithm used previously by the authors (Johnson and McDonald 2004a) for vortex motion near a single gap in an infinite wall is also used here. A further modification to the algorithm is necessary in this 


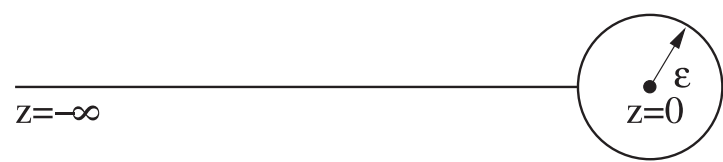

FIG. 5. Semi-infinite plate with a circle of radius $\varepsilon$ centered at $z=0$.

work since the current passes around the tip of the plate where the unsteady velocity field becomes singular. To preclude this singularity, a circular "exclusion zone" of small radius is centered on the plate tips $z= \pm L$. During the advection process, if a node on the contour enters the exclusion zone, it is projected onto the rim of the small circle, thus avoiding the singular regions near $z= \pm L$. The contour is then renoded to ensure its smoothness. Various radii for the exclusion zone were tested, leading to the conclusion that using a radius of 0.1 seems to have little qualitative effect on the dynamics. This "exclusion" procedure is tested explicitly in the next subsection for the special case of a semiinfinite plate with a circular tip: a boundary shape which can be constructed "exactly" using contour dynamics.

\section{a. Semi-infinite plate}

Before tackling the case of a finite width gap, the evolution of a coastal current around a single semi-infinite barrier stretching from $z=0$ to $\Re z=-\infty$ is studied. The steady solution is given by (9). This case is relevant to the flow of a coastal current about a sharp cape.

To incorporate the semi-infinite plate in contour dynamics, the usual method employed by the authors of finding an irrotational flow field correction that when added to that computed using contour dynamics gives zero normal velocity on the plate is used (see, e.g., Johnson and McDonald 2004b, 2005, 2007). In this case, computing the irrotational flow field involves a conformal map from the region surrounding the plate to the exterior of the unit circle. A further reason for studying this example is that there is, in fact, a map from the exterior of the plate with a circular tip of radius $\varepsilon$ in the $z$ plane (see Fig. 5) to the exterior of the unit circle in the $\zeta$ plane:

$$
\zeta=\frac{z^{1 / 2}+z-\epsilon}{z^{1 / 2}-z+\epsilon}
$$

This enables the exclusion zone method described above to be explicitly tested. That is, the approximation when nodes are projected back onto a circle of radius 0.1 at the tip of a straight barrier can be tested by comparing with a semi-infinite barrier of exactly the shape of a plate augmented by a circle of radius 0.1 by choosing $\varepsilon=0.1$ (as in Fig. 5) in (10). Such a test was carried out and little qualitative difference was observed in comparing the results-the conclusion being that the exclusion zone
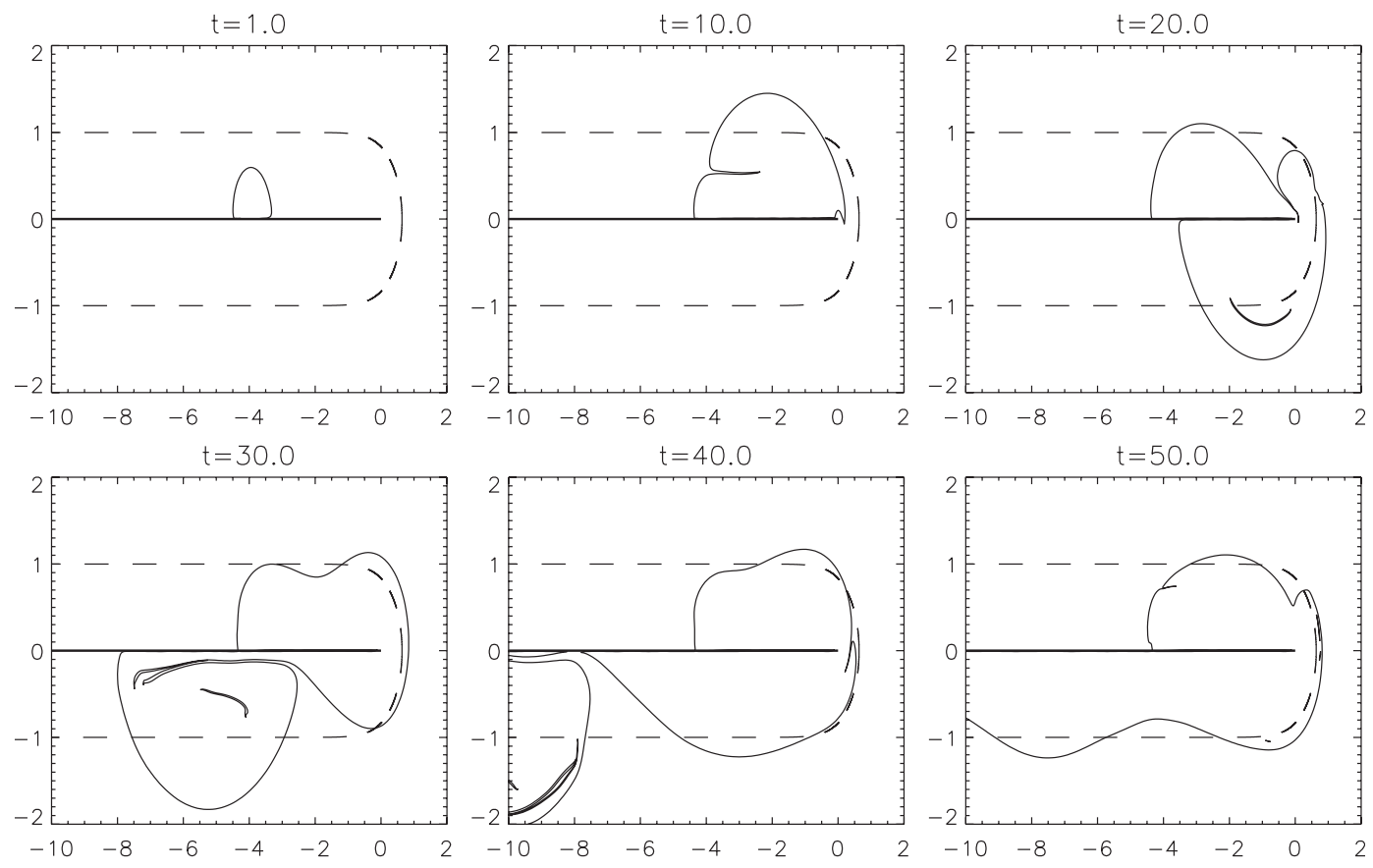

FIG. 6. Evolution of a current about a semi-infinite plate originating from a source at $z=-4$. The dashed line is the exact solution of Howison and King (1989). 

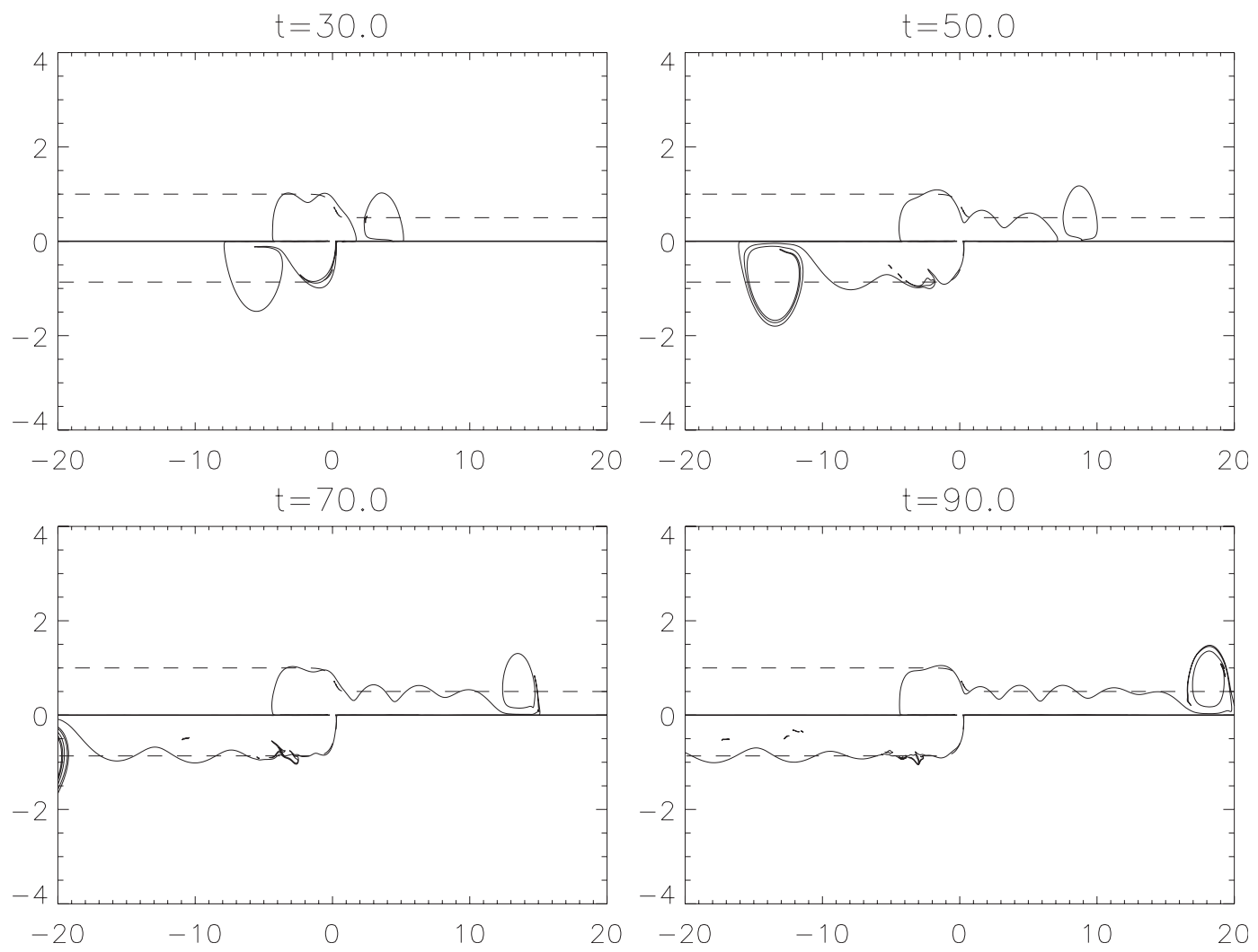

FIG. 7. Evolution of a current through a gap originating from a source at $z=-4$, with $\beta=0.5$ and $L=0.19$. The dashed line is the corresponding steady solution derived in section 2.

method is a reasonable and efficient way of treating the singularities occurring at the plate tips.

Figure 6 shows the evolution of the current about the plate. All vortical fluid emitted by the source eventually propagates around the tip and propagates toward $\Re z \rightarrow$ $-\infty$ on the opposite side to the barrier to the source. For large times it is evident that the shape of the current approaches the exact steady state (9). It is noteworthy that no distinct eddies are detached from the current during its evolution.

\section{b. Gap-leaping currents}

Figure 7 shows the evolution of a coastal current starting at $z=-4$ for across-gap flux $\beta=0.5$ and a gap of width $L=0.19$ [this being, from (7), the corresponding gap width for a steady solution]. The large eddy forming ahead of the current is typical for vortical currents (see, e.g., Stern and Pratt 1985; An and McDonald 2004; Johnson and McDonald 2006) and is observed here for both currents that leap across and those that pass through the gap. Behind these eddies, the current widths approach the equivalent steady solution (the dashed line) as time increases. This suggests that the steady solutions of the previous section are physically realizable and are also stable in this region of parameter space.

Figure 8 shows the evolution of a coastal current starting at $z=-4$ for across-gap flux $\beta=0.25$ and a gap of width $L=0.26$ [this being, from (7), the corresponding gap width for a steady solution]. In comparison to the previous example, the flux across the gap is smaller and the notable difference is that the across-gap current is manifested as a chain of eddies, with the lead eddy becoming completely detached and, owing to its relatively large size and hence circulation, propagating away from the chain of smaller eddies. In contrast, the current that forms from fluid passing through the gap matches well with the steady solution. There is some complicated folding of the contour, partly owing to the fact that velocity on the edge of the current vanishes and perturbations on the contour are therefore slow to propagate away.

\section{c. Zero flux through the gap}

In the previous examples a nonzero net flux though the gap was specified. In many situations, however, it is more natural to impose zero net flux through the gap, this being the situation when the basin in the lower half 

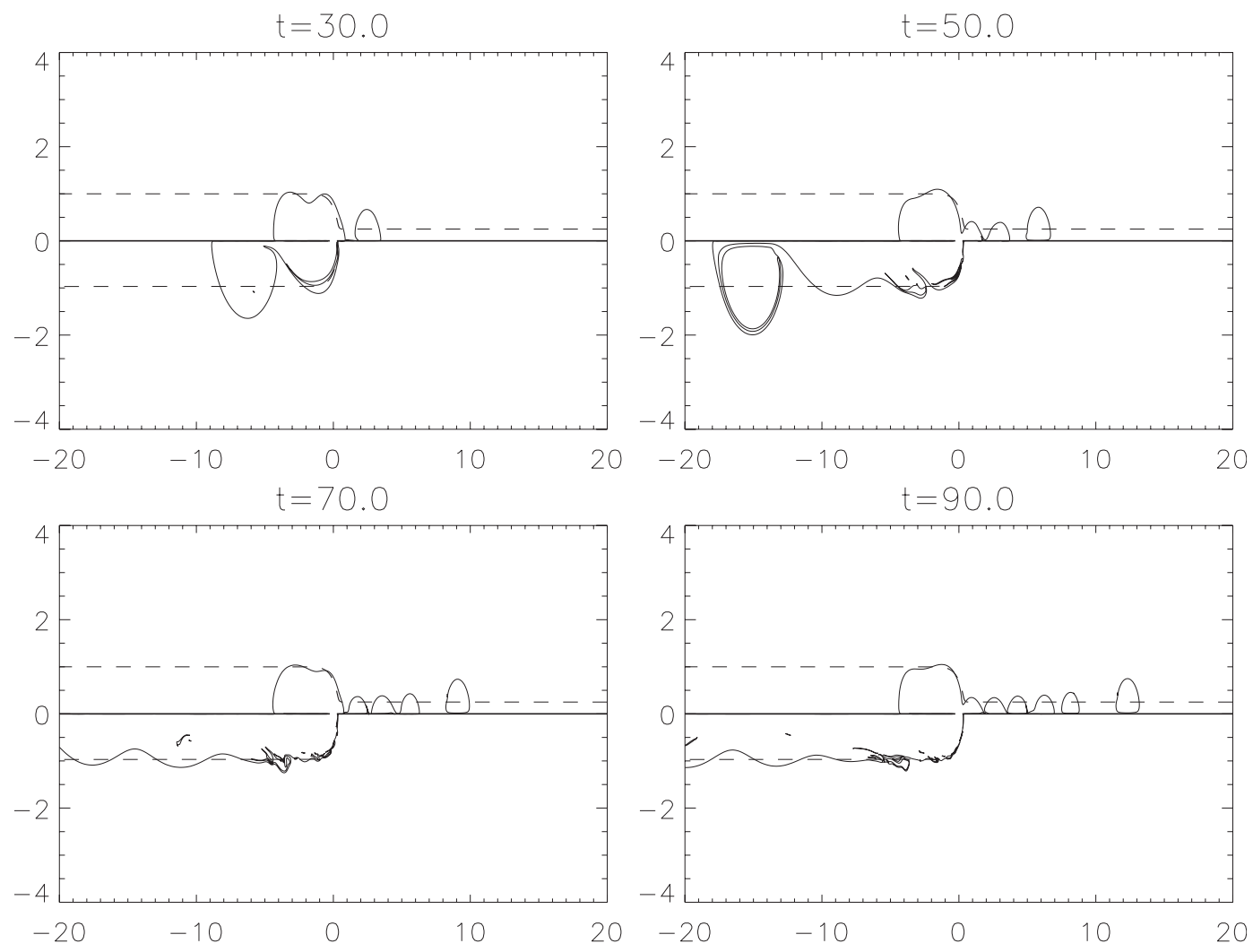

FIG. 8. As in Fig. 7, but with $\beta=0.25$ and $L=0.26$.

plane is finite. In this case the theory of section 2 is no longer applicable because the velocity outside the vortical current (i.e., the irrotational velocity field) does not necessarily vanish since there must be a return flow of nonvortical fluid from the lower to upper sides of the barrier. However, it is still possible to perform numerical experiments, and in the following examples the net flux is set to zero and the gap width is chosen.

Figure 9 shows the evolution of the current for a gap of width $L=0.2$. In this case a small amount of vortical fluid is able to penetrate the gap, but the majority leaps across the gap and evolves in a similar way to a current flowing along an infinite unbroken barrier (e.g., Stern and Pratt 1985; Johnson and McDonald 2006). Note that there is a thin layer of irrotational fluid separating the downstream coastal current from the barrier. This represents a nonzero flux of irrotational fluid through the gap from the lower half plane to the upper half plane and is required to reduce the net flux to zero.

Figure 10 shows the evolution of the current for a gap of larger width $L=0.8$. In this case a significant portion of the vortical fluid passes through the gap forming a well-defined coastal current on the opposite side of the barrier to the source. In order for the net flux to vanish there must be an equal and opposite flux of irrotational fluid. This return flux causes the vortical fluid leaping across the gap to be displaced upward - this fluid taking the form of a large eddy shedding event. Such eddy shedding as fluid crosses the gap is typical in these zeronet flux experiments for $L \geqslant 0.4$; the amount of fluid going into forming the eddies depends on the across-gap flux. In contrast, the through-gap fluid forms a welldefined uniform current whose flux becomes steady as time increases. This is illustrated in Fig. 11, which shows the through-gap flux of vortical fluid for various gap widths $L$ as a function of time. In a typical experiment the flux of vortical fluid through the gap is zero until the current reaches the gap at about $t=8$. The flux then increases rapidly, reaching a peak at about $t=18$. This peak corresponds to the formation of the large eddy at the head of the current on the opposite side of the barrier to the source. After some further transient behavior, the flux then settles down to an almost constant value. As $L$ increases, so does the flux of vortical fluid passing through the gap. Recall that the flux from the source is 0.5 so that for $L=1.0$ most of the vortical fluid passes through the gap. 

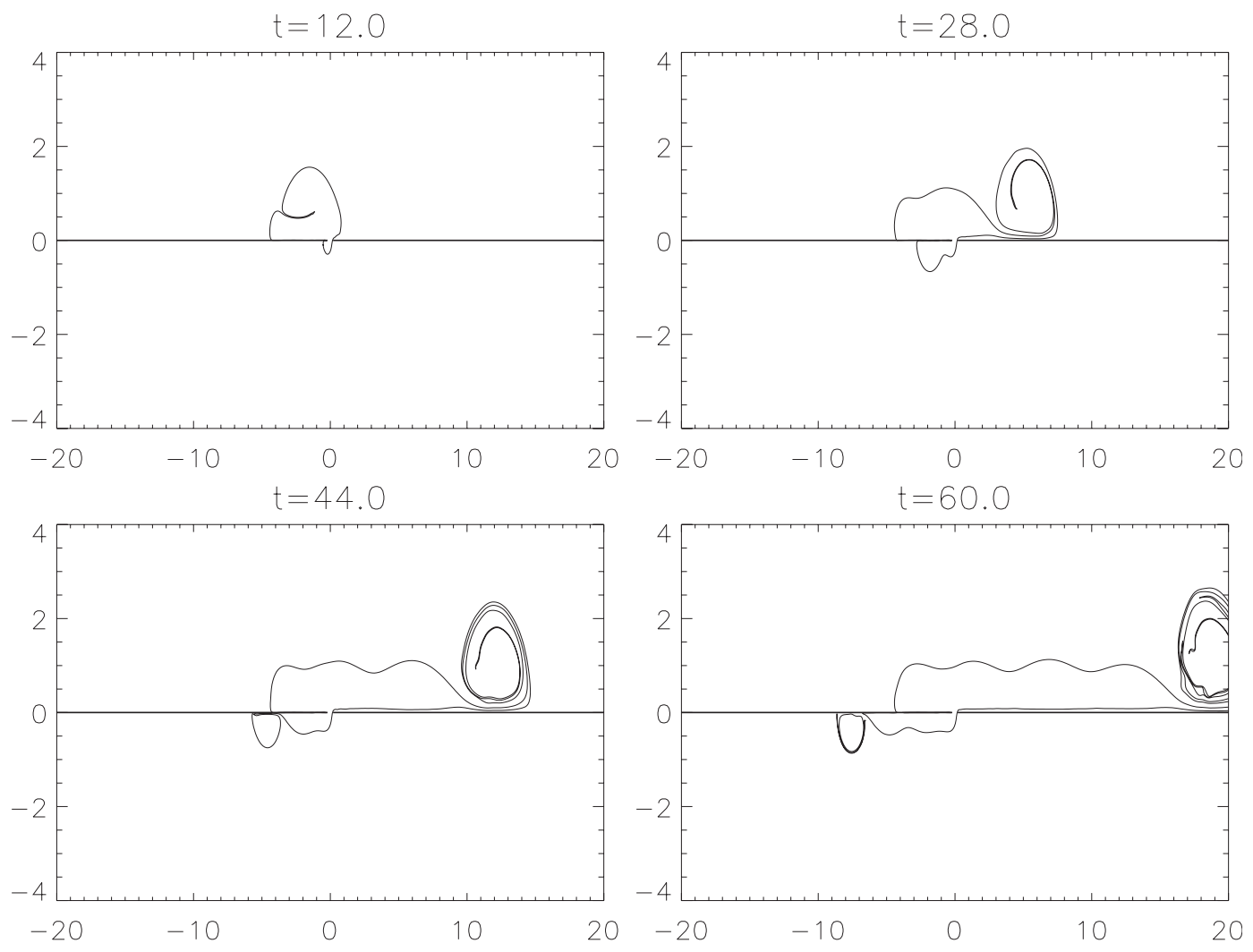

FIG. 9. As in Fig. 7, but with zero net flux and $L=0.2$.

\section{Discussion}

A family of exact solutions describing the bifurcation of a boundary current with constant vorticity near a gap has been found. It is shown numerically that timedependent flows initialized from a source of constant strength upstream of the gap approach the exact steady solutions in the large time limit. This suggests that the properties of the exact steady solutions are robust and may well be observable in the ocean. Some physical processes that are important in oceanic flows through gaps (e.g., baroclinicity, mixing, local bottom topography) have been neglected. Nevertheless, the simplified dynamics has enabled identification of generic behavior in the behavior of boundary currents near a gap separating two ocean basins. For instance, specifying the gap width or flux through the gap completely determines the structure of a steady current bifurcating at the gap. When the flux across the gap is sufficiently small, the across-gap transport is manifested as a chain of propagating eddies rather than a steady current.

The exact solution derived here, in addition to being useful for checking results of large-scale numerical models, can be used to predict the flux of the coastal current that approaches and flows through Unimak Pass. This region was also considered by Nof and Im (1985), who used an equivalent barotropic model of a buoyancy-driven coastal current and predicted that all of the current should pass through the gap. They cited observational evidence supporting their prediction.

The width of the pass at its narrowest point is $20 \mathrm{~km}$ and the upstream breadth of the current is $40 \mathrm{~km}$ (i.e., twice the gap width; Nof and Im 1985). The present theory immediately gives, using Fig. 4, a nondimensional flux across-gap flux of 0.124 or $25 \%$ of the upstream flux, implying that $75 \%$ of mass flux of the current passes through the gap in comparison to the $100 \%$ predicted by Nof and Im (1985). This prediction made by the theory in this paper seems reasonable, bearing in mind that observations show that the current is unsteady and has a baroclinic structure with prominent outcropping of isopycnals (e.g., Schumacher et al. 1982; Stabeno et al. 2002); such effects are not included in this work.

For the situation in which there is no net flux through the gap, numerical experiments show that, as the gap width increases, so does the proportion of the vortical current passing through the gap. At large times the transport in this current approaches a steady value, whereas the current that leaps across the gap remains 
$t=12.0$
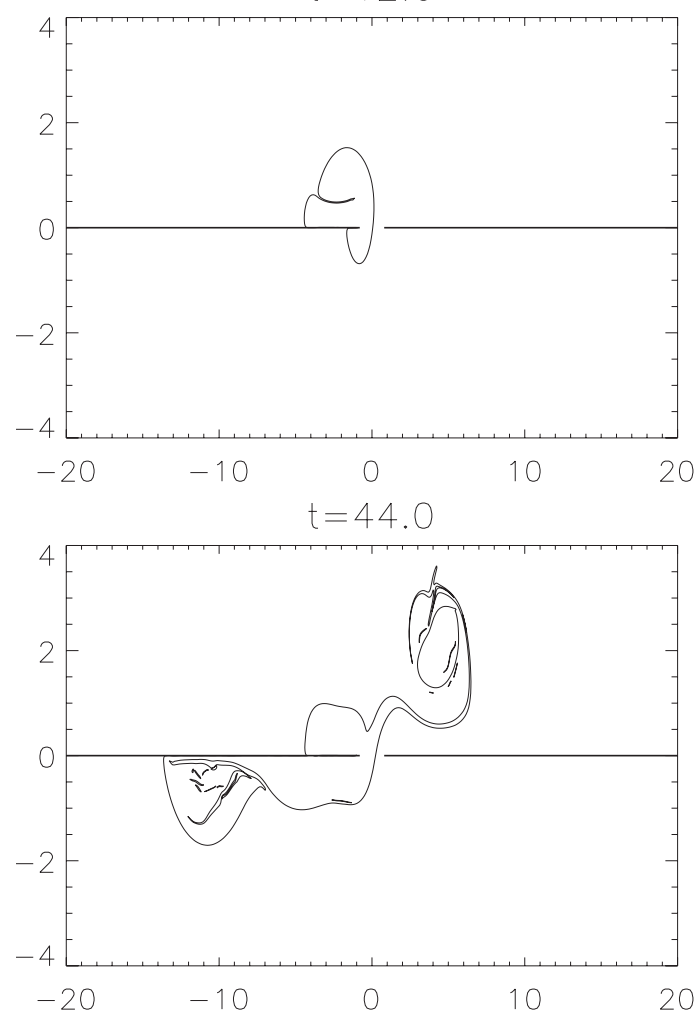

$t=28.0$
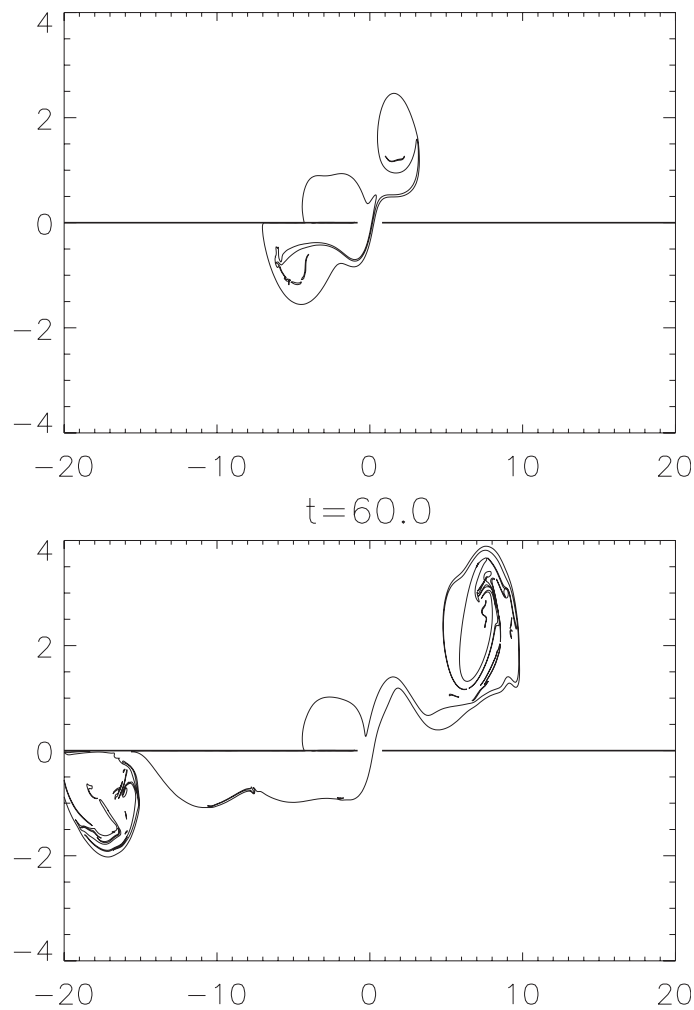

FIG. 10. As in Fig. 9, but for $L=0.8$.

unsteady, reflecting the generation of eddies at the gap for the range of $L$ tested. For $L \geq 1$, at large times, virtually all the vortical fluid passes through the gap. A current propagating around the tip of a semi-infinite wall (i.e., a cape) is able to do so without forming eddies. This behavior differs from the eddying found by (Pichevin and Nof 1996) for the dynamically different equivalentbarotropic flow around a semi-infinite barrier.

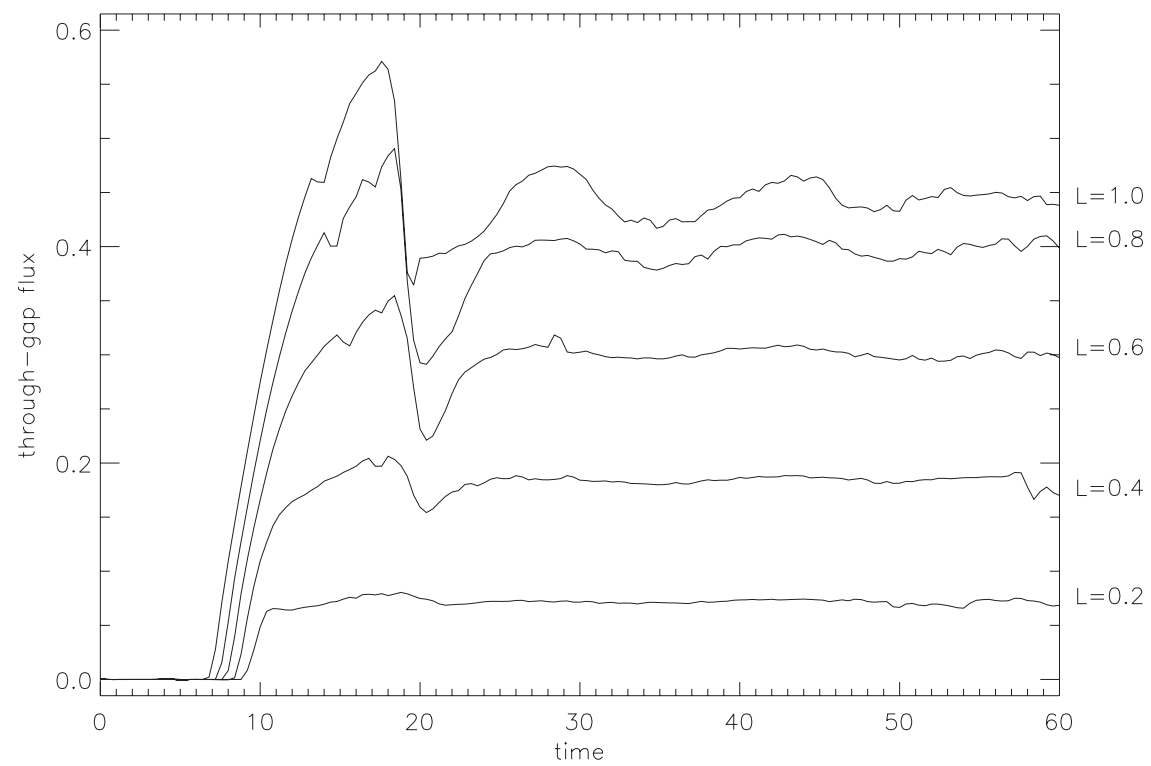

FIG. 11. Time-dependent through-gap fluxes of vortical fluid for various gap widths in the case of zero net flux through the gap. 
Acknowledgments. The authors are grateful for the useful comments made by the reviewers.

\section{REFERENCES}

An, B. W., and N. R. McDonald, 2004: Coastal currents driven by outflow and vorticity and their interaction with topography. Cont. Shelf Res., 24, 1531-1547.

— and - 2005: Coastal currents and eddies and their interaction with topography. Dyn. Atmos. Oceans, 40, 237-253.

Cherubin, L., X. Carton, and D. G. Dritschel, 1996: Vortex expulsion by a zonal coastal jet on a transverse canyon. Proc. Second Int. Workshop on Vortex Flows, Paris, France, SMAI, 101-121.

Crowdy, D. G., 1999: A class of exact multipolar vortices. Phys. Fluids, 11, 2556-2564.

—_, and J. Marshall, 2006: The motion of a point vortex through gaps in walls. J. Fluid Mech., 551, 31-48.

— J. Fluid Mech., 593, 235-254.

Herbaut, C., F. Codron, and M. Crepon, 1998: Separation of a coastal current at a strait level: Case of the Strait of Sicily. J. Phys. Oceanogr., 28, 1346-1362.

Howison, S. D., and J. R. King, 1989: Explicit solutions to six freeboundary problems in fluid flow and diffusion. IMA J. Appl. Math., 42, 155-175.

Johnson, E. R., and N. R. McDonald, 2004a: The motion of a vortex near a gap in a wall. Phys. Fluids, 16, 462-469.

$\longrightarrow$, and,$- 2004 \mathrm{~b}$ : The motion of a vortex near two circular cylinders. Proc. Roy. Soc. London A, 460, 939-954.

, and $\_, 2005$ : Vortices near barriers with multiple gaps. J. Fluid Mech., 531, 335-358.
, and _ 2006: Vortical source-sink flow against a wall: The initial value problem and exact steady states. Phys. Fluids, 18, 076601, doi:10.1063/1.2221353.

_ , and -2007 : Vortical flow around a finite plate. Quart. J. Mech. Appl. Math., 60, 65-72.

Kubokawa, A., 1991: On the behaviour of outflows with low potential vorticity from a sea strait. Tellus, 43A, 168-176.

Nof, D., and S. H. Im, 1985: Suction through broad oceanic gaps. J. Phys. Oceanogr., 15, 1721-1732.

Pedlosky, J., 2001: The transparency of ocean barriers to Rossby waves: The Rossby slit problem. J. Phys. Oceanogr., 31, 336-352.

Pichevin, T., and D. Nof, 1996: The eddy cannon. Deep-Sea Res., 43, 1475-1507.

Pratt, L., and M. E. Stern, 1986: Dynamics of potential vorticity fronts and eddy detachment. J. Phys. Oceanogr., 16, 11011120.

— , and M. A. Spall, 2003: A porous-medium theory for barotropic flow through ridges and archipelagos. J. Phys. Oceanogr., 33, 2702-2718.

Schumacher, J. D., C. A. Pearson, and J. E. Overland, 1982: On exchange of water between the Gulf of Alaska and the Bering Sea through Unimak Pass. J. Geophys. Res., 87, 5785-5795.

Sheremet, V. A., 2001: Hysteresis of a western boundary current leaping across a gap. J. Phys. Oceanogr., 31, 1247-1259.

_ , and J. Kuehl, 2007: Gap-leaping western boundary current in a circular tank model. J. Phys. Oceanogr., 37, 1488-1495.

Stabeno, P. J., R. K. Reed, and J. M. Napp, 2002: Transport through Unimak Pass, Alaska. Deep-Sea Res. II, 49, 5919-5930.

Stern, M. E., and L. Pratt, 1985: Dynamics of vorticity fronts. J. Fluid Mech., 161, 513-532.

Timmermans, M. L., P. Winsor, and J. A. Whitehead, 2005: Deepwater flow over the Lomonosov Ridge in the Arctic Ocean. J. Phys. Oceanogr., 35, 1489-1493. 